\title{
Standalone operation of an EGOFET for ultra-sensitive detection of HIV
}

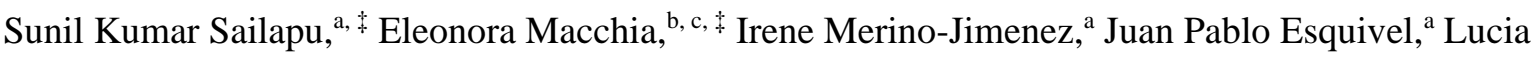 \\ Sarcina, ${ }^{\mathrm{b}}$ G. Scamarcio, ${ }^{\mathrm{d}, \mathrm{e}}$ Shelley D. Minteer, ${ }^{\mathrm{f}}$ Luisa Torsi ${ }^{\mathrm{b}, \mathrm{c}, \mathrm{g}}$ and Neus Sabaté ${ }^{\mathrm{a}, \mathrm{h},{ }^{*}}$ \\ ${ }^{a}$ Instituto de Microelectrónica de Barcelona, IMB-CNM (CSIC), C/ del Til.lers, Campus UAB, 08193 \\ Bellaterra, Barcelona, Spain \\ ${ }^{\mathrm{b}}$ Department of Chemistry, Università degli Studi di Bari A. Moro 4, Via Orabona, 70126 Bari, Italy \\ 'The Faculty of Science and Engineering, Åbo Akademi University, 20500 Turku, Finland \\ d Dipartimento Interateneo di Fisica "M. Merlin" - Università degli Studi di Bari - "Aldo Moro" - Bari, Italy \\ ${ }^{\mathrm{e}}$ CNR - Istituto di Fotonica e Nanotecnologie, Unità di Bari, Italy \\ ${ }^{\mathrm{f}}$ Department of Chemistry, University of Utah, Salt Lake City, UT 84112, USA \\ ${ }^{\mathrm{g}}$ CSGI (Centre for Colloid and Surface Science), 70125 Bari, Italy \\ ${ }^{\mathrm{h}}$ Catalan Institution for Research and Advanced Studies (ICREA), P.L. Companys 23, 08010 Barcelona, Spain
}

${ }^{\ddagger}$ S. K. Sailapu and E. Macchia equally contributed to this work

*neus.sabate@imb-cnm.csic.es

\begin{abstract}
A point-of-care (POC) device to enable de-centralized diagnostics can effectively reduce the time to treatment, especially in case of infectious diseases. However, many of the POC solutions presented so far do not comply with the ASSURED (affordable, sensitive, specific, user-friendly, rapid and robust, equipment free, and deliverable to users) guidelines that are needed to ensure their on-field deployment. Herein, we present the proof of concept of a self-powered platform that operates using the analysed fluid, mimicking a blood sample, for early stage detection of HIV-1 infection. The platform contains a smart interfacing circuit to operate an ultra-sensitive electrolyte-gated field-effect transistor (EGOFET) as a sensor and facilitates an easy and affordable readout mechanism. The sensor transduces the biorecognition event taking place at the gate electrode functionalized with the antibody against the HIV-1 p24 capsid protein, while it is powered via paper-based biofuel cell (BFC) that extracts the energy from the analysed sample itself. The self-powered platform is demonstrated to achieve detection of HIV-1 p24 antigens in fM range, suitable for early diagnosis. From these developments, a cost-effective digital POC device able to detect the transition from "healthy" to "infected" state at single-molecule precision, with no dependency on external power sources while using minimal components and simpler approach, is foreseen.
\end{abstract}

\section{Keywords}

Bioelectronics; Biofuel cell; Electrolyte-gated organic field-effect transistor; HIV-1 p24 detection; Selfpowered bioelectronic devices 


\section{Introduction}

Widespread diagnosis is crucial for early detection and treatment, particularly in case of infectious diseases such as human immunodeficiency virus (HIV), tuberculosis, malaria, Ebola etc., that have been leading causes of death in resource limited locations (Maffert et al. 2017). For instance, an early detection of p24 antigens that make up the HIV viral core would ensure proper antiretroviral therapy for effective immune response and prevent vulnerable opportunistic infections (Kahn and Walker 1998). The host serum in the first acute phase of HIV infection contains from 10 to 3000 virions $\mathrm{mL}^{-1}$, with an estimated p24 concentration of around $50 \mathrm{aM}$ to $15 \mathrm{fM}$ (Rissin et al. 2010). Though, polymerase chain reaction based ribonucleic acid test (Adler et al. 2008; Hecht et al. 2002) has proven to be a suitable diagnostic tool in the first acute phase, the need for expensive equipment and reagents highly impact their global outreach (Barlow et al. 1997; Kosaka et al. 2017). Moreover, the approach often involves a tedious routine of sample extraction and operation in suitable laboratories for analysis. This together with the involved cost, resources and the long assay procedures does not comply with ASSURED (affordable, sensitive, specific, user-friendly, rapid and robust, equipment free, and deliverable to users) characteristics defined by the World Health Organization (Maffert et al. 2017; Plate and Rapid 2007) towards the objective of delivering required diagnostic aid to resource-limited settings.

Thereby, different sensing strategies towards obtaining point-of-care (POC) devices for a rapid, highly sensitive response and quantitative digital information through a simple operation have been assessed in the last few years. Techniques based on enzyme-linked immune sorbent assay (ELISA) employing Au nanoparticles (Lee et al. 2004) and DNA barcodes (Tang and Hewlett 2010) have significantly pushed the HIV-1 p24 detection limit to less than $0.5 \mathrm{pg} \mathrm{mL}^{-1}$. Even better results have recently been acquired with molecularly imprinted polymer based electrochemical sensor (Ma et al. 2017). Specially promising are the bioelectronic label-free sensors based on an electrolyte-gated organic field-effect transistor (EGOFET) (Tobjörk and Österbacka 2011; Torsi et al. 2013). EGOFETs are fieldeffect transistors that involve an ionic conducting electrolyte as dielectric and connects the transducing gate electrode (optionally detachable) with an organic semiconductor-based channel. With effective capacitive coupling between gate/electrolyte and electrolyte/semiconductor interfaces, these offer excellent sensitivities and sub-volt operation that could be potentially achieved with low power sources like fuel cells and batteries. These are endowed with selectivity via the integration of suitable biorecognition elements and have lately exhibited sensitivities capable of single molecule detection (Macchia et al. 2018a; Macchia et al. 2018b; Tobjörk and Österbacka 2011). The key to this SingleMolecule detection with a Transistor or SiMoT (Macchia et al. 2018a), is the immobilization of trillions of capturing antibodies on a millimeter-sized gate. The extremely large number of capturing sites covalently attached to a large interface, addressed as wide-field sensing approach (Gooding and Gaus 2016), increases the interaction cross-section with the biomarker to be detected, allowing the assay of 
extremely diluted solutions. At the same time, a cooperative effect, enabled by a hydrogen-bonding network and fuelled through operating gate voltage, amplifies the impact of a single or of few bindings, that can be detected as a sizable variation of the transistor channel current (Macchia et al. 2018a; Macchia et al. 2019a). Recently, the SiMoT approach has been proposed also for detection of HIV-1 p24 at the single molecule limit (Macchia et al. 2019b). This disruptive technology that promises a simple operation offers biosensing capabilities comparable to state-of-the-art label-free single-molecule sensors based on nano-scale devices. Additionally, in contrast to the nanotechnology-based systems, the SiMoT platform can be fabricated via solution-processed scalable procedures that are foreseen to be developed into a low-cost, large-area printed technology (Tobjörk and Österbacka 2011). However, despite salient features that make SiMoT platforms as promising candidates for ASSURED POC devices, no efforts have been made so far towards the integration of these sensing components to demonstrate a portable standalone device operation without the need of dedicated electronic equipment.

Herein, we present a self-powered platform containing a smart interfacing circuit for the operation of an ultra-sensitive bioelectronic sensor that allows selective detection of the HIV-1 p24 antigen at 1 $\mathrm{fM}$ concentration with a hand-held multimeter. The platform involves the use of minimal and low-cost electronic components, and achieves self-powered operation by using a compact paper-based glucose biofuel cell (BFC) that is able to activate with a small volume $(3.5 \mu \mathrm{L})$ of the biological sample being analysed (Fig. 1). The current approach is a significant step forward to the standalone POC bioelectronic devices with relevant opportunities for ultrasensitive detection of biomarkers, that could have a terrific impact on the public health.

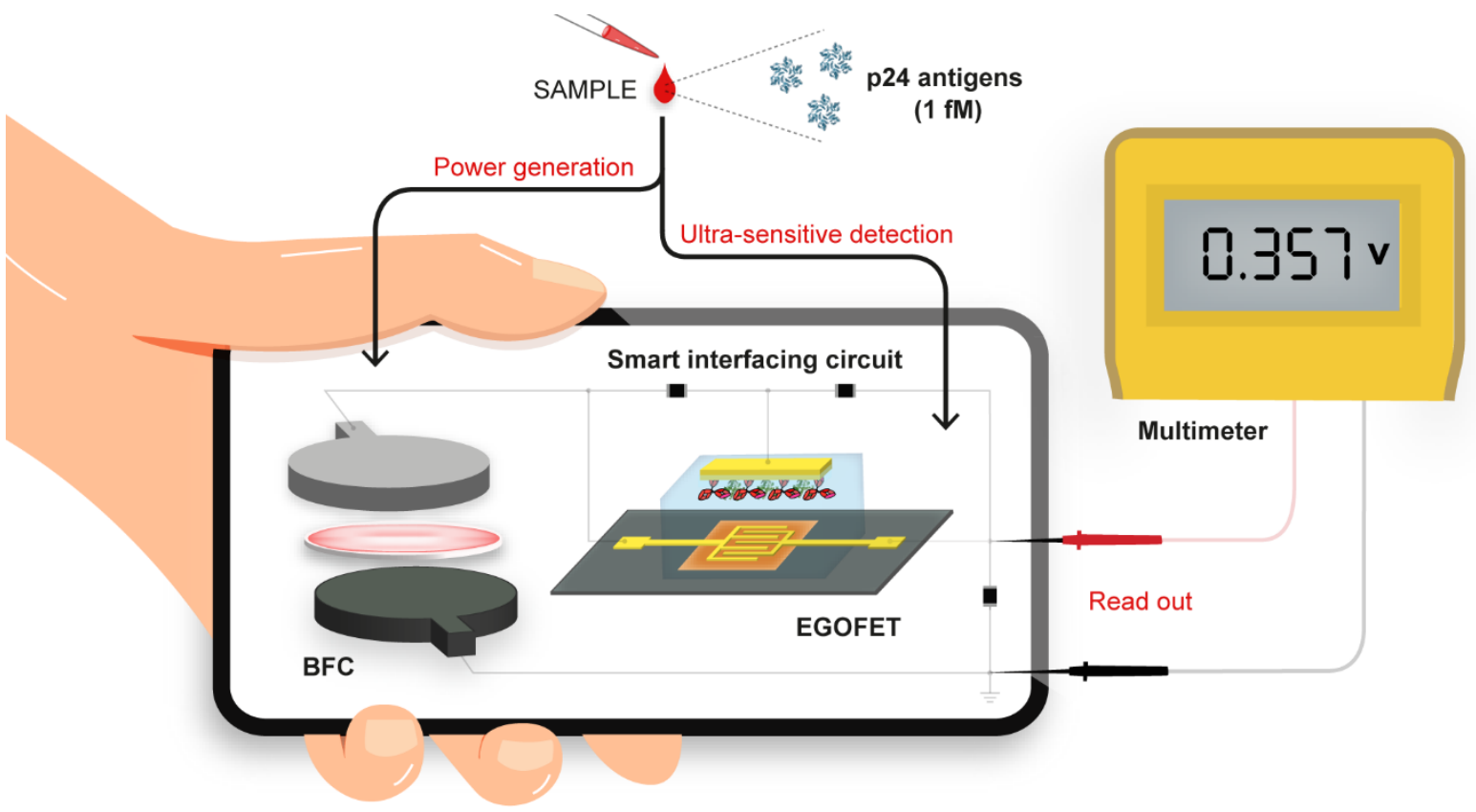

Fig. 1. Schematic illustration of the approach towards development of an ultrasensitive self-powered bioelectronic platform. The self-powered platform performed operation of the EGOFET through conjugating a paper-based BFC that is activated from the sample being analyzed. A suitable interfacing circuit promoted an on-demand detection of the biomarkers and easy readout using a handheld multimeter. 


\section{Material and Methods}

The details about the materials used, fabrication and operation of EGOFET, bio-functionalization of the gate electrode, design and assembly of paper-based BFC, electrodes and electrolyte preparation, paperbased BFC operation, self-powered diagnostic platform and the estimation of current through it are present in the Supplementary Information.

\section{Results and Discussion}

\section{1. p24 antigen sensing response analysis of EGOFET using a semiconductor parameter analyzer}

The detection platform for HIV-1 p24 protein rely on a Poly(3-hexylthiophene-2,5-diyl), P3HT organic semiconductor based EGOFET (Fig. 2a) that consisted an Au gate tagged with the capturing probe antiHIV-1 p24. With water (HPLC grade deionised) as gating electrolyte, the output characteristics (Fig. 2b) of typical EGOFET, measured using a bare Au gate, revealed drain current ( $\mathrm{I}_{\mathrm{D}}$ ) modulation with linear and active regions for the applied drain voltages $\left(\mathrm{V}_{\mathrm{DS}}\right)$ at a given gate potential $\left(\mathrm{V}_{\mathrm{GS}}\right)$. A negligible hysteresis of $I_{D}$ was showed by the EGOFET when $V_{D S}$ was cycled in increasing and decreasing values. Also from the characteristics, field-effect based operation at biasing voltages less than $1 \mathrm{~V}\left(\mathrm{~V}_{\mathrm{DS}}, \mathrm{V}_{\mathrm{GS}}\right)$ with $I_{D}$ in the order of hundreds of $n A$ to tens of $\mu \mathrm{A}$ is clearly evident. However, in order to operate the EGOFET as a gate voltage controlled current source (active region), $\mathrm{V}_{\mathrm{DS}}$ was fixed at $-0.5 \mathrm{~V}$ during measurement of transfer curves in the subsequent steps.

As a proof of concept, the detection of a $1 \mathrm{fM}$ concentration of HIV-1 p24 proteins was targeted in development of the screening assay that suits to asses an individual in the initial phase of the infection, where the estimated concentration of p24 proteins is known to be between 50 aM to $15 \mathrm{fM}$. For this, the surface of the bare Au gate as shown in Fig. 2a was bio-functionalised with anti-HIV-1 p24 antibodies, through a chemical self-assembled monolayer (chem-SAM). To minimize non-specific interactions, the gate was further packed with bovine serum albumin (BSA) to fill the spaces left after the conjugation. The transfer curves measured using this bio-functionalized gate, after its incubation in PBS standard-solutions containing $1 \mathrm{fM} \mathrm{HIV}-1 \mathrm{p} 24$ proteins, indicated a change in $\mathrm{I}_{\mathrm{D}}$ from the reference curve obtained prior using the same gate incubated in only PBS (Fig. 2c). However, when measurements were taken for similar experiments with a solely BSA functionalized Au gate electrode to capture HIV$1 \mathrm{p} 24$ proteins, no such variation in $\mathrm{I}_{D}$ was observed (Fig. 2d). It may be noted that BSA do not bind to HIV-1 p24, thus serves as a negative control experiment. The transfer characteristic curves also indicated a shift in threshold voltage $\left(\mathrm{V}_{\mathrm{T}}\right)$ due to the bio-functionalization when compared to that acquired using a bare Au gate as reported previously (Macchia et al. 2018a). In case of anti-HIV-1 p24 / BSA functionalized Au gate, a $\mathrm{V}_{\mathrm{T}}$ of $-0.20 \pm 0.05 \mathrm{~V}$ was observed, while a $\mathrm{V}_{\mathrm{T}}$ of $-0.12 \pm 0.05 \mathrm{~V}$ was measured with the bare Au gate. Thus, it can be inferred from the transfer curves that the EGOFET's response is specific to the antigen-antibody interaction of HIV-1 p24 proteins. 
(a)

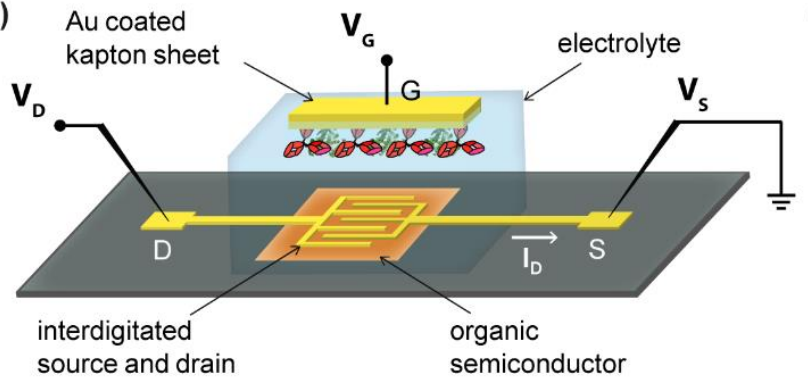

(b)

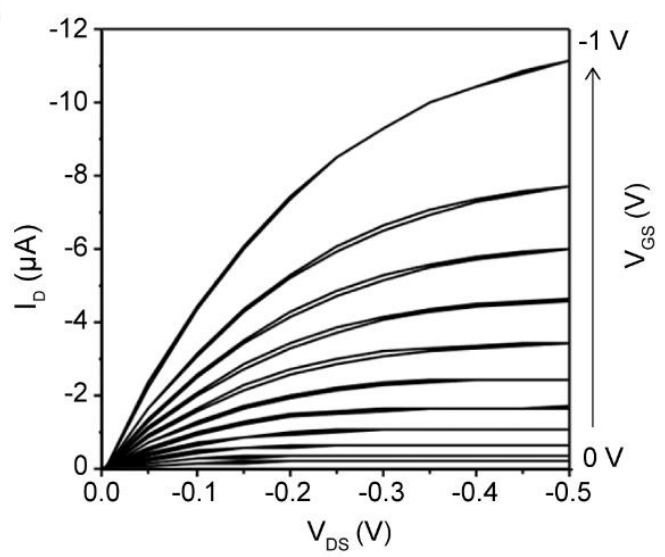

(c)

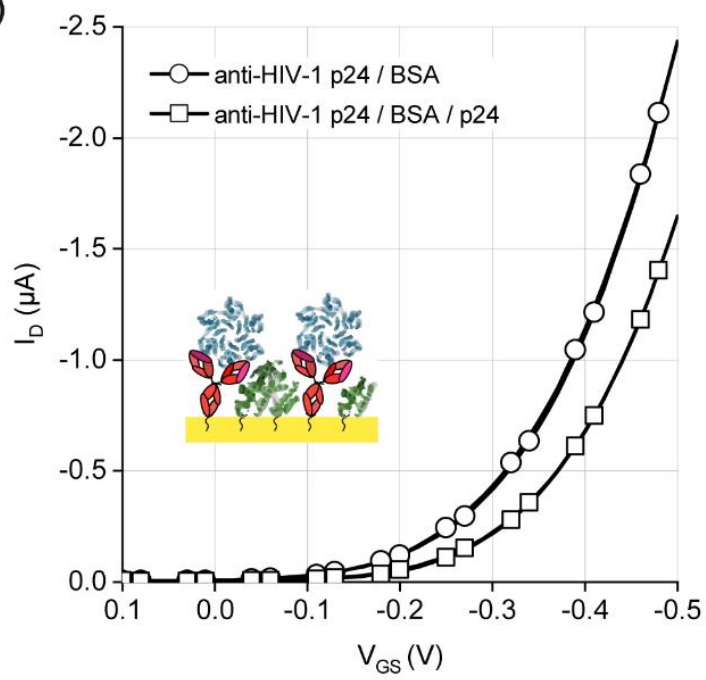

BSA

(d)

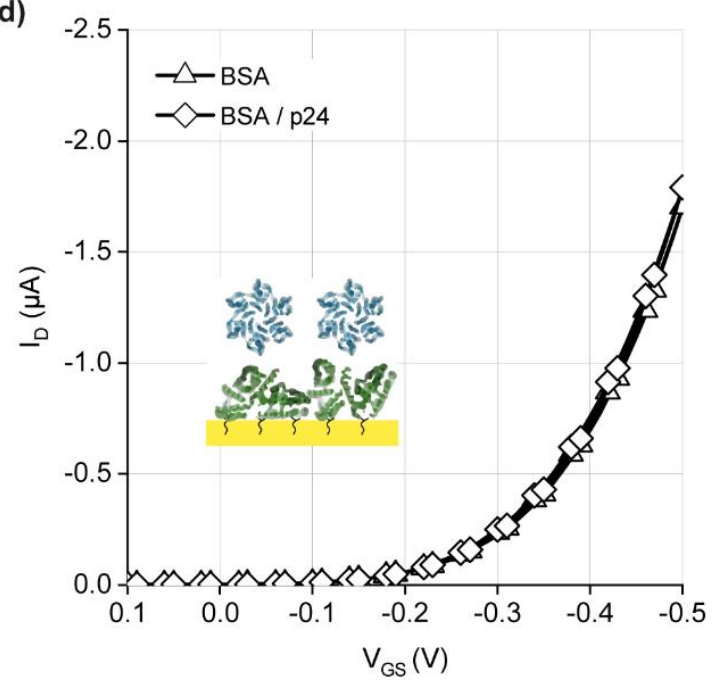

Fig. 2. (a) The three-dimensional schematic structure of the EGOFET employed for HIV-1 p24 antigen detection along with the sketches of the proteins used. (b) Output characteristics $\left(\mathrm{I}_{\mathrm{D}} v s . \mathrm{V}_{\mathrm{DS}}\right.$ ) of the fabricated EGOFET measured using a bare $\mathrm{Au}$ gate. (c, d) Transfer characteristics $\left(\mathrm{I}_{\mathrm{D}} v s . \mathrm{V}_{\mathrm{GS}}\right.$ at $\left.\mathrm{V}_{\mathrm{DS}}=-0.5 \mathrm{~V}\right)$ for anti-HIV-1 p24 / BSA and solely BSA functionalized Au gate before and after the interaction with HIV-1 p24 antigens (1 fM). A distinct change in characteristics was observed in case of anti-HIV-1 p24 / BSA functionalized Au gate upon specific interaction with HIV-1 p24 antigens.

The relative current change in $I_{D}$ upon exposure of the gate electrode to the target analyte in the PBS solutions is typically used as the sensing response (Eq.1),

$$
\text { Sensing Response }=\frac{\Delta \mathrm{I}}{\mathrm{I}_{\mathrm{o}}}=\frac{\mathrm{I}-\mathrm{I}_{\mathrm{o}}}{\mathrm{I}_{\mathrm{o}}}
$$

where $I_{0}$ and I denote the current values obtained before and after the exposure to the sample solution. It was observed that the response obtained with an anti-HIV-1 p24 / BSA functionalized Au gate was clearly distinct in comparison to the solely BSA functionalized Au gate electrode (Fig. 3a). The overall response change between them is given by Eq. 2, 


$$
\text { Responsechange }(\%)=\left(\frac{\Delta \mathrm{I}_{\mathrm{a}}}{\mathrm{I}_{\mathrm{oa}}}-\frac{\Delta \mathrm{I}_{\mathrm{b}}}{\mathrm{I}_{\mathrm{ob}}}\right) \times 100
$$

where subscript a, b corresponds to measurements with solely BSA and anti-HIV-1 p24 / BSA functionalized Au gate electrodes respectively. As further indicated by the characteristics in Fig. 3b, the biosensor showed a $50 \%$ difference in response between the two bio-functionalised gates at $\mathrm{V}_{\mathrm{GS}}$ values between $-0.1 \mathrm{~V}$ and $-0.3 \mathrm{~V}$, which is a wide operational zone of sensing that provides robustness in terms of designing suitable interfacing electronics.
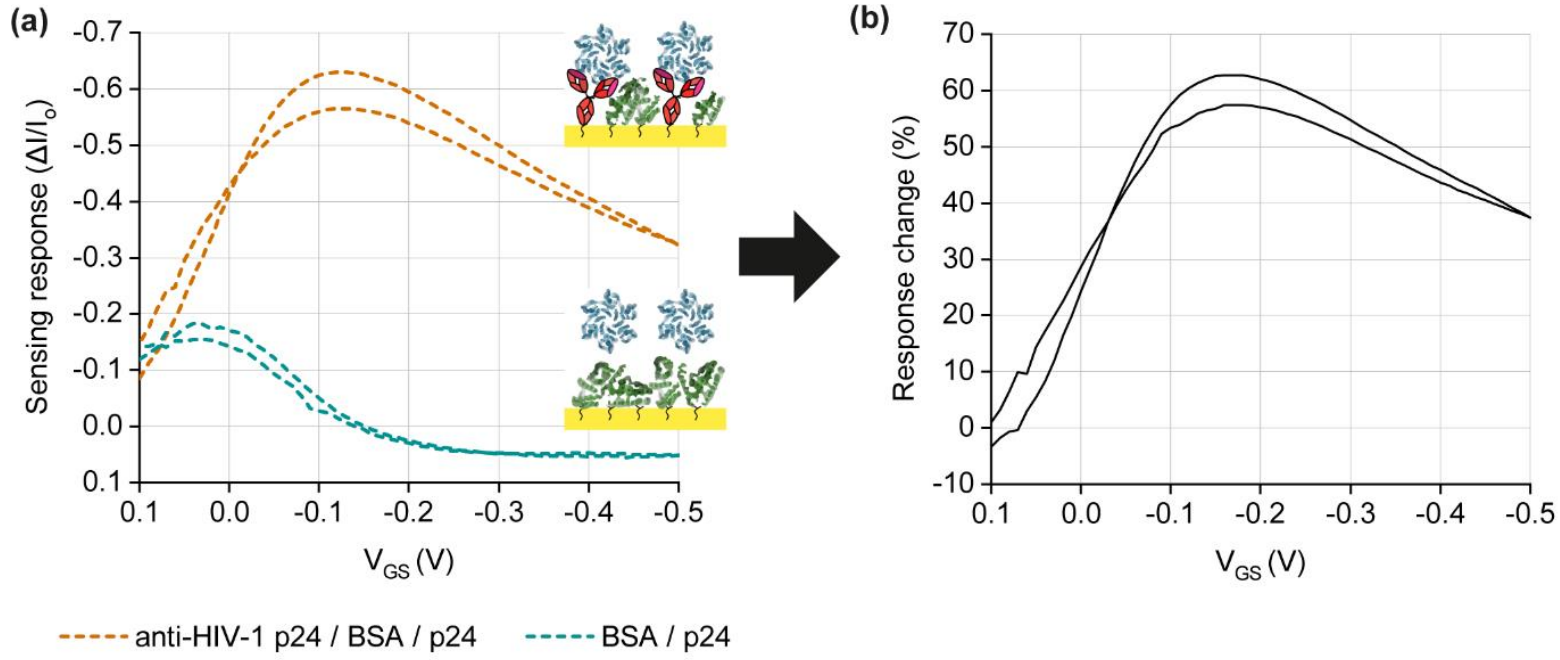

Fig. 3. (a) The response curve indicating the relative changes in $I_{D}\left(\Delta I / I_{0}\right)$ for a series of $V_{G S}$ at $V_{D S}=-0.5 V$ upon exposure to HIV-1 p24 protein (1 fM) in case of anti-HIV-1 p24 / BSA and solely BSA functionalized Au gate electrode when biased using an external power supply. (b) The overall change in the sensing response obtained between solely BSA and anti-HIV-1 p24 / BSA functionalized Au gates, at different $\mathrm{V}_{\mathrm{GS}}$ (for $\mathrm{V}_{\mathrm{DS}}=-0.5 \mathrm{~V}$ ) for the detection of $1 \mathrm{fM}$ concentration of HIV-1 p24 protein.

\subsection{Smart interfacing circuit for BFC-EGOFET integration}

The driving of the EGOFET has been assessed by designing a smart interfacing circuit (Fig. 4a) to bias all the sensing steps (described in section 3.1). Unlike the traditional approach involving scanning of $\mathrm{V}_{\mathrm{GS}}$, as performed with a semiconductor parameter analyzer to obtain the transfer curves (Fig. 2c and d), the interfacing circuit operates the EGOFET at a fixed $V_{G S}, V_{D S}$ and yields a specific output voltage readable by a handheld multimeter without the need for complex instrumentation. Moreover, the circuit uses the power generated by a glucose fuel cell to set the EGOFET at the operating point and provide the required current for stable operation.

Hence, it was essential to initially derive this optimum operating voltage for $\mathrm{V}_{\mathrm{GS}}$ and $\mathrm{V}_{\mathrm{DS}}$. We selected the value of $\mathrm{V}_{\mathrm{GS}}$ to be $-0.3 \mathrm{~V}$ based on two criteria: (i) it has to be a voltage lower than all the threshold voltages recorded along the different sensing steps (the minimum value of $\mathrm{V}_{\mathrm{T}}$ corresponded to $-0.2 \mathrm{~V}$ in case of HIV-1 p24 functionalized Au gate) and (ii) it has to provide a response change of at least $50 \%$ between the two bio-functionalized gates. (Fig. 3). The open circuit voltage (OCV) of BFC, 
employing FAD-GDH as the primary constituent of the anode and silver oxide as cathode was measured to be about $0.85 \mathrm{~V}$ when fed with a PBS solution containing $5 \mathrm{mM}$ glucose as electrolyte (generally accepted as the average glucose content in blood under fasting condition). As shown in Fig. 4a, if $V_{B F C}$ is considered to be the voltage supplied by the BFC, then the required $\mathrm{V}_{\mathrm{GS}}$ (given by Eq. 3) of $-0.3 \mathrm{~V}$ was achieved by connecting a voltage divider formed through resistors $R_{1}$ and $R_{2}$, selected as $1.8 \mathrm{M} \Omega$ and 3.3 $M \Omega$ respectively. $R_{1}$ and $R_{2}$ were set to be within the range of $M \Omega$ to minimize the current demand of the voltage divider to the BFC.

$$
\mathrm{V}_{\mathrm{GS}}=-\mathrm{V}_{\mathrm{BFC}}\left(\frac{\mathrm{R}_{1}}{\mathrm{R}_{1}+\mathrm{R}_{2}}\right)
$$

$\mathrm{V}_{\mathrm{DS}}$ can be easily applied by connecting the BFC terminals directly across the drain and source terminals of EGOFET $\left(\mathrm{V}_{\mathrm{BFC}}=-\mathrm{V}_{\mathrm{DS}}\right)$. However, in order to extract a measurable output voltage $\left(\mathrm{V}_{\text {out }}\right)$ corresponding to the $I_{D}$ flowing through the EGOFET, we incorporated a series resistor, $R_{D}$. The addition of $R_{D}$ will introduce variations in $V_{D S}$ that depend on the $I_{D}$ flowing through the EGOFET conducting channel during different sensing steps (see Eq. 4).

$$
\mathrm{V}_{\mathrm{DS}}=-\left(\mathrm{V}_{\mathrm{BFC}}+\mathrm{I}_{\mathrm{D}} \mathrm{R}_{\mathrm{D}}\right)
$$

Therefore, despite the anticipated deviations in $\mathrm{V}_{\mathrm{DS}}$ through placing of $\mathrm{R}_{\mathrm{D}}$, any influence in the output current $I_{D}$ can be avoided by keeping EGOFET operational voltages in the active region, where the sensing parameter $I_{D}$ is tuned only by $V_{G S}$ (which is already fixed at $-0.3 \mathrm{~V}$ ). This condition is reached when $V_{D S} \leq V_{D S(s a t)}$, for all the steps involved in the sensing operation, being $V_{D S(s a t)}=V_{G S}-$ $\mathrm{V}_{\mathrm{T}}$. In the current experiment, the maximum value recorded for $\mathrm{V}_{\mathrm{T}}$ was $-0.12 \mathrm{~V}$ (in case of bare $\mathrm{Au}$ gate) and therefore, considering $\mathrm{V}_{\mathrm{DS} \text { (sat) }}$ to be at around -0.18 V ensures the EGOFET operation in active region for all the sensing steps involved in the measurement protocol. In order to keep $V_{D S}$ value below $-0.18 \mathrm{~V}$, it is desirable to ensure that the voltage drop across $\mathrm{R}_{\mathrm{D}}$ at the highest magnitude of drain current $\left(I_{D(\max )}\right)$ is kept below a certain value. Considering the transfer curves in Fig. $2 c$ and d obtained for different steps, the maximum magnitude of drain current recorded when characterizing the EGOFET with the semiconductor parameter analyser was observed to be $\sim-435 \mathrm{nA}$ (with anti-HIV-1 p24 functionalized $\mathrm{Au}$ gate). In order to avoid boundary operation, we provided a safety margin of about $100 \%$, and decided to set $\mathrm{I}_{\mathrm{D}(\max )}$ value to $-900 \mathrm{nA}$ (twice the anticipated current). This limited the $\mathrm{R}_{\mathrm{D}} \leq$ $745 \mathrm{k} \Omega$ (from Eq. 4) to bias the EGOFET at an operational voltage of $\mathrm{V}_{\mathrm{DS}} \leq-0.18 \mathrm{~V}\left(\mathrm{~V}_{\mathrm{DS}(\mathrm{sat})}\right)$. However, for the present case, the operating point of $\mathrm{V}_{\mathrm{DS}}(-0.4 \mathrm{~V})$ was chosen to be well beyond the active region threshold ( $\mathrm{V}_{\mathrm{DS} \text { (sat) }}$ ), which set the value of $\mathrm{R}_{\mathrm{D}}$ to be $500 \mathrm{k} \Omega$. Importantly, the value of $\mathrm{R}_{\mathrm{D}}$ in the order of $\mathrm{k} \Omega$ allows to generate voltage readouts across it in the $\mathrm{mV}$ range, that can be easily measured with a handheld multimeter. Thereby, the circuit not only interfaces for standalone operation of EGOFET, but 
further generates a convenient method of measuring the output. This greatly simplifies the application of EGOFET and makes it more amicable for POC platforms.

\subsection{Characterization of paper-based BFC}

(a)

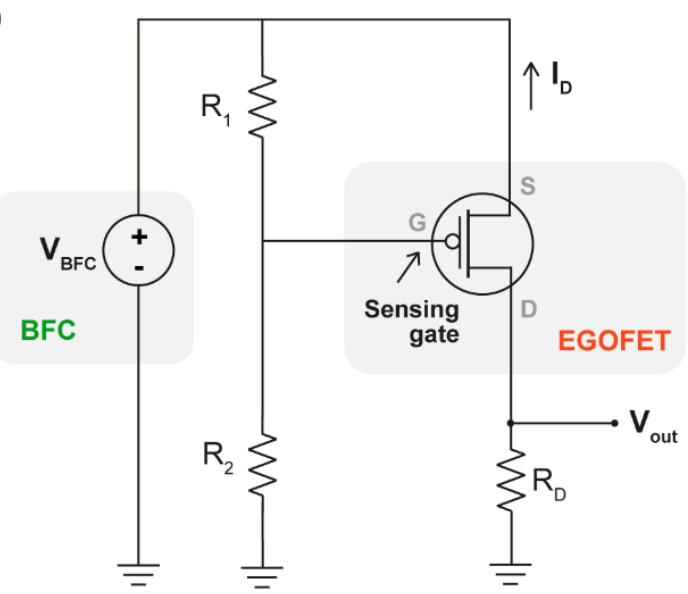

(c)

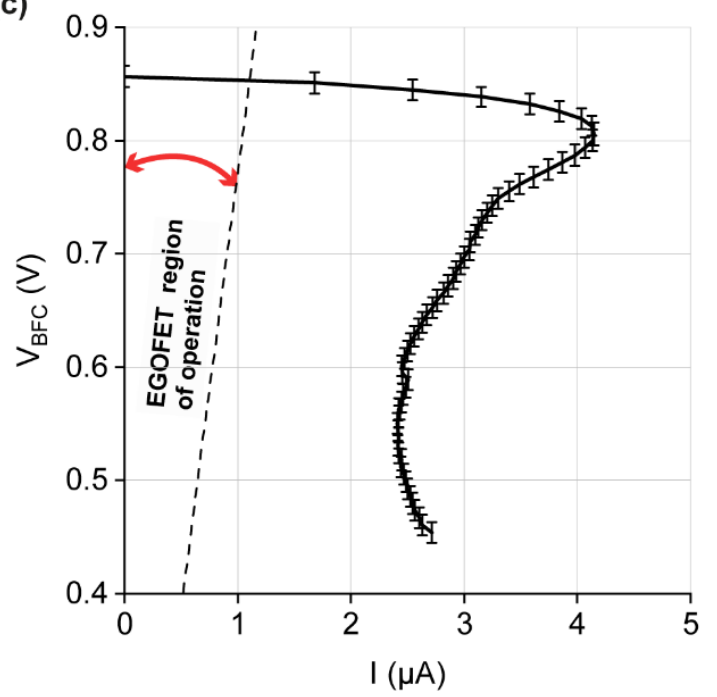

(b)

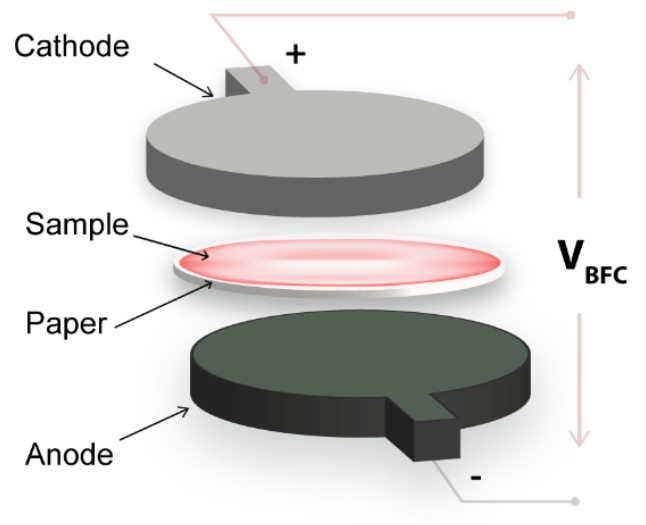

(d)

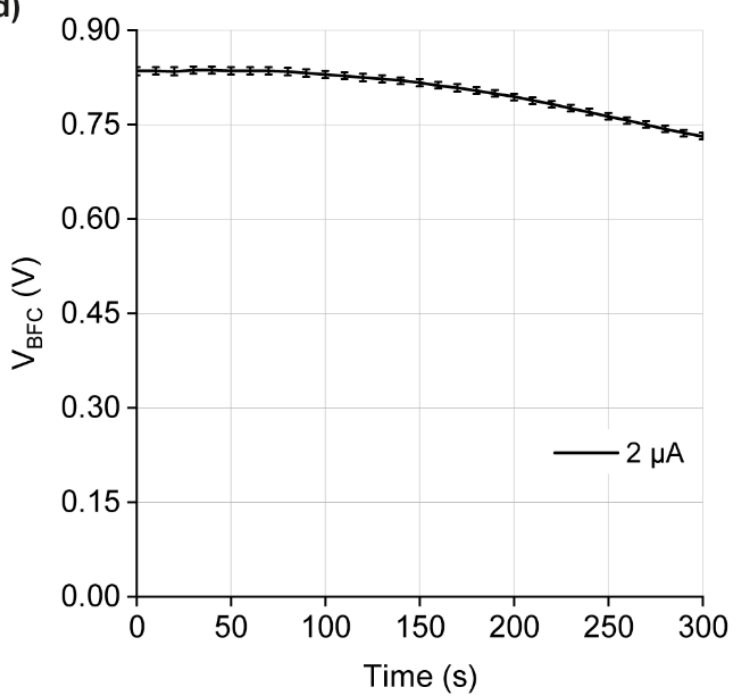

Fig. 4. (a) Interfacing circuit for standalone operation of EGOFET with paper-based BFC for HIV-1 p24 detection. (b) Scheme illustrating the structure of paper-based BFC used for standalone operation of the EGOFET. The power was generated from the analyzed sample absorbed on paper, that is sandwiched between screen-printed anode and cathode electrodes. (c) Polarization curve of paper-based BFC operated with a PBS solution containing $5 \mathrm{mM}$ glucose solution at a scan rate of $1 \mathrm{mV} \mathrm{s}^{-1}$. The potential across BFC was approximately around $0.845 \mathrm{~V}$ at the maximum operating current of $1.1 \mu \mathrm{A}$ required for EGOFET operation. (d) Voltage across paper-based BFC operated with a PBS solution of $5 \mathrm{mM}$ glucose while suppling a current of $2 \mu \mathrm{A}$ (chronopotentiometry curve). The data in (c, d) are represented as an average with respective standard deviations of measurements made using three independent, identical paper-based BFCs.

The paper-based BFC was developed considering the voltage and current requirements for the independent operation of EGOFET. The paper-based BFC proposed here is intended to be prospectively fed with the same biological sample that is to be analysed, which releases the need of connecting the platform to an external power supply and renders the system to be completely autonomous. Due to the 
limited available volume of sample to perform the test (blood drop coming from a finger prick ranges from $0.3 \mu \mathrm{L}$ to $50 \mu \mathrm{L}$ ), the BFC was developed to operate with a minimum volume of $3.5 \mu \mathrm{L}$ of sample solution. The cell design parameters, choice and concentration of electrode materials were optimised to obtain the required power levels from this limited amount of sample. The BFC with a sandwich type layout (Fig. 4b), used $5 \mathrm{mM}$ glucose solution (contained in PBS) as electrolyte that was placed on a glass-fiber-paper. An open circuit voltage (OCV) of about $0.85 \mathrm{~V}$ was generated by the paper-based BFC. Additionally, the polarization curve showed a peak current of $4.15 \pm 0.11 \mu \mathrm{A}$ and a maximum power of $3.36 \pm 0.06 \mu \mathrm{W}$ (Fig. 4c). Taking into account the maximum current expected to flow across the transistor channel and the equivalent impedance of the voltage divider, we estimated that the entire circuit would require the paper-based BFC to provide a stable current of at least $1.1 \mu \mathrm{A}$ (refer to Estimation of current for operation of the self-powered platform in SI, Fig. S1). According to the polarization curve, the potential across BFC corresponding to this current was expected to be approximately around $0.845 \mathrm{~V}$. Thereby, it was evident from the OCV and the polarization curve that the paper-based BFC is capable of supplying the required voltage in the range of EGOFET operation. The effect on the performance of BFC for other extreme concentrations of glucose, say 2.5 and $10 \mathrm{mM}$ (corresponding to hypoglycaemia and hyperglycaemia conditions) were also analysed using the polarization curves. The changes $(\Delta \mathrm{V})$ in supply voltage $\left(\mathrm{V}_{\mathrm{BFC}}\right)$ from the OCV at these concentrations were found to be identical below the required maximum current of $1.1 \mu \mathrm{A}$ (Fig. S2). This showed the BFC's ability to operate at a wide range of glucose concentrations commonly observed in patients. The voltage stability of BFC over time was examined through chronopotentiometry measurements (Fig. 4d) while delivering a current of about $2 \mu \mathrm{A}$. It was observed that the $\mathrm{BFC}$ maintained a stable voltage with deviation less than $0.001,0.005$ and $0.01 \mathrm{~V}$ for at least 75,100 and $120 \mathrm{~s}$ respectively. The influence of this voltage drop on the operating voltage $\mathrm{V}_{\mathrm{GS}}$ of EGOFET was found to be less than $0.005 \mathrm{~V}$ (from Eq. 3 ) in the initial $120 \mathrm{~s}$. The transfer characteristics of EGOFET (Fig. 2c, d) indicated that any deviation caused in $\mathrm{V}_{\mathrm{GS}}$ within $0.01 \mathrm{~V}$ (caused from a $\mathrm{V}_{\mathrm{BFC}}$ drift of $0.028 \mathrm{~V}$ ) from the operating point of $-0.3 \mathrm{~V}$ results in a response change (from Eq. 2) of $48 \%$ and higher between the two bio-functionalized gates. Further, for $300 \mathrm{~s}$ of continuous operation that caused a maximum drift of $-0.04 \mathrm{~V}$ in $\mathrm{V}_{\mathrm{GS}}$, a response change of at least 32\% can still be assured to detect HIV-1 p24 antigen despite a maximum deviation of $0.1 \mathrm{~V}$ in $\mathrm{V}_{\mathrm{BFC}}$.

However, when EGOFET is biased, its response is quick ( $<1 \mathrm{sec}$ ) and the output voltage appears rather instantaneously. So, during any of the sensing steps, it is enough to acquire a single output voltage measurement within the first few seconds (preferably before $120 \mathrm{~s}$ ) to register the bio-interaction occurred on the gate surface. This interval is more than convenient to acquire a digital measurement easily with a simple multimeter.

\subsection{Standalone operation of EGOFET for detection of HIV-1 p24}


The proof-of-concept of a self-powered diagnostic platform was built connecting the paper-based BFC and the EGOFET through the smart interfacing circuit. A photograph of the setup is featured in Fig. 5a. The sensing measurements were performed by monitoring $I_{D}$ and $V_{\text {out }}$ values using the anti-HIV-1 p24 / BSA functionalized Au gate, before and after exposure to a $1 \mathrm{fM}$ concentration of HIV-1 p24 protein solution (Fig. 5b and c). Similar set of negative control experiments were also carried out using the $\mathrm{Au}$ gate functionalized with solely BSA (Fig. $5 \mathrm{~d}$ and e). Indeed, very stable voltages (and currents) were acquired and a distinct change in $\mathrm{V}_{\text {out }}$ (and $\mathrm{I}_{\mathrm{D}}$ ) was recorded for the control and sensing experiments. Further, the measured voltages in the range of $\mathrm{mV}$ were easily obtained with a handheld multimeter. As $R_{D}$ is a fixed value, the sensing response (Eq. 1) and the response change (Eq. 2) can be calculated directly by substituting the measured $V_{\text {out }}$ in place of $I_{D}$ for the respective sensing step. The relative changes $\left(\Delta \mathrm{V} / \mathrm{V}_{\mathrm{o}}\right)$ in $\mathrm{V}_{\text {out }}$ upon exposure to the target marker during standalone operation of EGOFET returned values of $-0.46 \pm 0.03$ and $0.04 \pm 0.02$ in case of HIV-1 p24 / BSA and solely BSA functionalized $\mathrm{Au}$ gates respectively. Here, $\mathrm{V}_{\mathrm{o}}$ and $\mathrm{V}$ correspond to voltage measured across $\mathrm{R}_{\mathrm{D}}$ before and after the exposure to the analyte solution. The $\Delta \mathrm{V} / \mathrm{V}_{\mathrm{o}}$ values obtained in standalone operation were in good agreement with the data gathered using semiconductor parameter analyser when EGOFET was biased externally (Fig. 5f). Moreover, despite the variations in recorded output voltages for different EGOFETs, the interfacing circuit allowed to decipher the differences in response (Fig. S3). The observed results further proved the very high response reproducibility of the proposed platforms. 
(a)

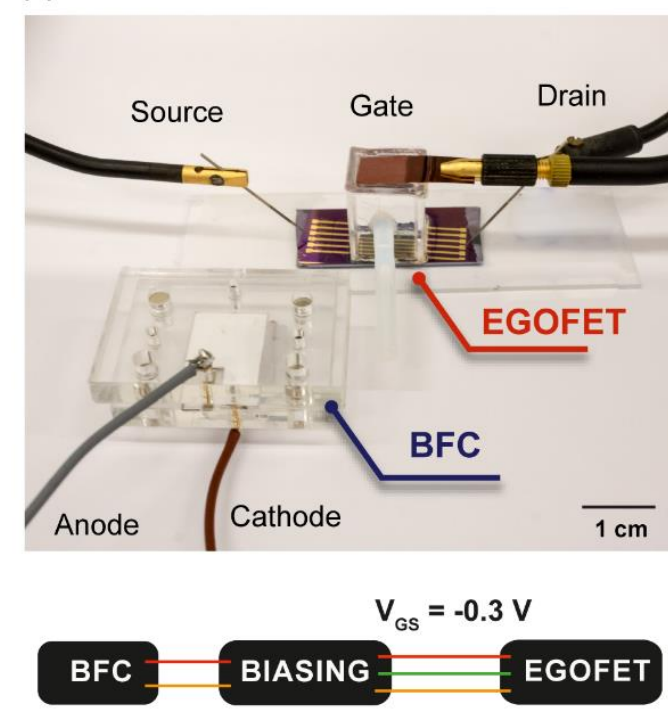

(b)

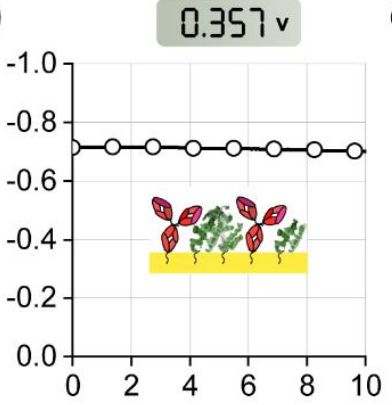

胥

(d)

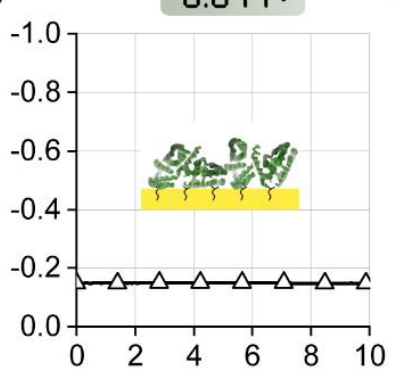

(c) $0.200 \mathrm{v}$

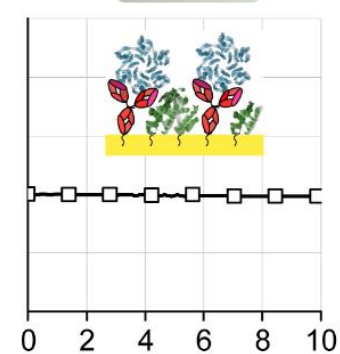

(e)

$0.076 \mathrm{v}$

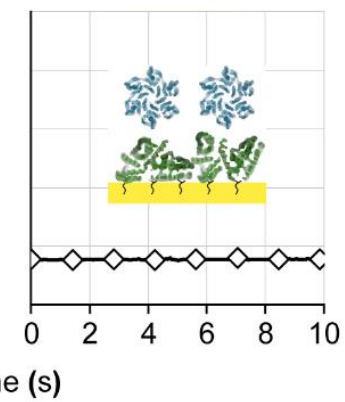

- - anti-HIV1 p24 / BSA

$\triangle-B S A$

$\rightarrow-$ anti-HIV1 p24 / BSA / p24

$\prec$ BSA / p24

(f)

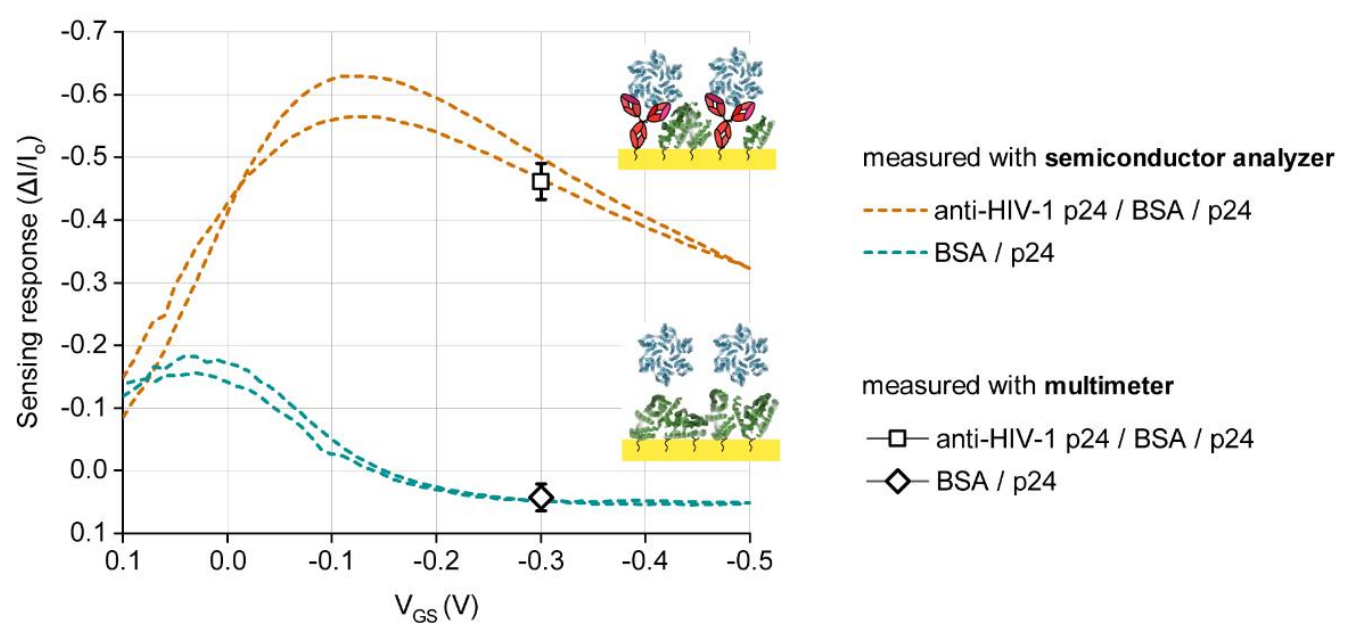

Fig. 5. (a) Photograph shows the operation of EGOFET connected to a paper-based BFC through an interfacing circuit for detection of biomarker, HIV-1 p24 (1 fM). (b, c) $V_{\text {out }}, I_{D}$ values measured during self-powered operation with BFC, along different steps of sensing. A relative change in $\mathrm{V}_{\text {out }}$ of around 50\% was observed with an antiHIV-1 p24 / BSA functionalized Au gate, before and after exposure to PBS standard solutions of HIV-1 p24 protein $(1 \mathrm{fM}) .(\mathrm{d}, \mathrm{e})$ Voltages obtained with solely BSA functionalized Au gate, before and after exposure to HIV-1 antigens showed no such change. (f) The overlap of the sensing responses $\left(\Delta V / V_{o}\right)$ of the EGOFET operated with $\mathrm{BFC}$ over the values measured previously when powered through an external source (for a series of $\mathrm{V}_{\mathrm{GS}}$ ) upon exposure to HIV-1 p24 antigen (1 fM). The data are represented as an average with the relevant relative-standard-deviation of measurements acquired with different EGOFET's and BFC's (reproducibility error). 


\section{Conclusions}

This work presents an approach to a standalone operation of an EGOFET through a self-powered smart sensing platform. The low operational voltages and currents required for EGOFET working allow the use of glucose BFC as the sole power source to drive it through a smart interfacing circuit that yields output voltages readable with a handheld multimeter. In fact, a small volume $(3.5 \mu \mathrm{L})$ of the analysed sample prove to be enough for the BFC to reliably deliver a stable output voltage for a sufficient time to acquire a digital measurement. Interestingly, the self-powered platform detects the presence of HIV$1 \mathrm{p} 24$ proteins at a concentration of $1 \mathrm{fM}$, and is promising for the POC based diagnosis of early stage HIV-1 infection. While bulky, complex and expensive equipment have been generally required to obtain EGOFET response based on drain current (in order of $\mathrm{nA}$ ), the circuit in the current work provides a output voltage (in $\mathrm{mV}$ range) that can be easily measured with a portable handheld multimeter to obtain similar results. The circuit is built with a minimum number of passive electronic components that present the capability of achieving a completely printable biosensing device applicable for the detection of a wide spectrum of clinically relevant biomarkers at an affordable cost. In a view to provide a decentralized approach to diagnostics, the proposed self-powered platform is a crucial development and could initiate relevant opportunities in the field of ultrasensitive POC testing, particularly in rural locations.

\section{Appendix A}

Electronic Supplementary Information

\section{Acknowledgement}

Cinzia Di Franco, Rosaria Anna Picca and Kyriaki Manoli are acknowledged for the fabrication and characterization of the electrolyte-gated organic field-effect transistor.

Sensore bio-elettronico usa-e-getta per l'HIV auto-alimentato da una cella a combustibile biologica (BioElSens\&Fuel) - Dottorati innovativi con caratterizzazione industriale - PON R\&I 2014-2020; Single molecule bio-electronic smart system array for clinical testing (SiMBiT) founded by the European Commission under H2020 program (GA - 824946); ERC Consolidator Grant (SUPERCELL - GA.648518); P-Sphere project (MSCA-COFUND-665919).

\section{References}

Adler, M., Wacker, R., Niemeyer, C.M., 2008. Analyst 133, 702-718.

Barlow, K.L., Tosswill, J.H., Parry, J.V., Clewley, J.P., 1997. J. Clin. Microbiol. 35, 2846-2853.

Gooding, J.J., Gaus, K., 2016. Angew. Chem. Int. Ed. Engl. 55, 11354-11366.

Hecht, F.M., Busch, M.P., Rawal, B., Webb, M., Rosenberg, E., Swanson, M., Chesney, M., Anderson, J., Levy, J., Kahn, J.O., 2002. AIDS 16, 1119-1129.

Kahn, J.O., Walker, B.D., 1998. N. Engl. J. Med. 339, 33-39.

Kosaka, P.M., Pini, V., Calleja, M., Tamayo, J., 2017. PLoS One 12, e0171899. 
Lee, K.-B., Kim, E.-Y., Mirkin, C.A., Wolinsky, S.M., 2004. Nano Lett. 4, 1869-1872.

Ma, Y., Shen, X.L., Zeng, Q., Wang, H.S., Wang, L.S., 2017. Talanta 164, 121-127.

Macchia, E., Manoli, K., Holzer, B., Di Franco, C., Ghittorelli, M., Torricelli, F., Alberga, D., Mangiatordi, G.F., Palazzo, G., Scamarcio, G., Torsi, L., 2018a. Nat Commun 9, 3223.

Macchia, E., Romele, P., Manoli, K., Ghittorelli, M., Magliulo, M., Kovács-Vajna, Z.M., Torricelli, F., Torsi, L., 2018b. Flex. Print. Electron. 3, 034002.

Macchia, E., Tiwari, A., Manoli, K., Holzer, B., Ditaranto, N., Picca, R.A., Cioffi, N., Di Franco, C., Scamarcio, G., Palazzo, G., Torsi, L., 2019a. Chem. Mater. 31, 6476-6483.

Macchia, E., Sarcina, L., Picca, R.A., Manoli, K., Di Franco, C., Scamarcio, G., Torsi, L., 2019b. Anal. Bioanal. Chem. DOI: 10.1007/s00216-019-02319-7.

Maffert, P., Reverchon, S., Nasser, W., Rozand, C., Abaibou, H., 2017. Eur. J. Clin. Microbiol. Infect. Dis. $36,1717-1731$.

Malhotra, U., Berrey, M.M., Huang, Y., Markee, J., Brown, D.J., Ap, S., Musey, L., Schacker, T., Corey, L., McElrath, M.J., 2000. J. Infect. Dis. 181, 121-131.

Plate, D.K., Rapid, H.I.V.T.E.W.G., 2007. AIDS Res. Hum. Retroviruses 23, 1491-1498.

Rissin, D.M., Kan, C.W., Campbell, T.G., Howes, S.C., Fournier, D.R., Song, L., Piech, T., Patel, P.P., Chang, L., Rivnak, A.J., Ferrell, E.P., Randall, J.D., Provuncher, G.K., Walt, D.R., Duffy, D.C., 2010. Nat. Biotechnol. 28, 595.

Tang, S., Hewlett, I., 2010. J. Infect. Dis. 201, S59-64.

Tobjork, D., Osterbacka, R., 2011. Adv. Mater. 23, 1935-1961.

Torsi, L., Magliulo, M., Manoli, K., Palazzo, G., 2013. Chem. Soc. Rev. 42, 8612-8628. 